This item was submitted to Loughborough's Research Repository by the author.

Items in Figshare are protected by copyright, with all rights reserved, unless otherwise indicated.

\title{
Towards a digital human representation in an industrial digital twin
}

PLEASE CITE THE PUBLISHED VERSION

https://ssrn.com/abstract=3717733

PUBLISHER

SSRN

VERSION

AM (Accepted Manuscript)

\section{PUBLISHER STATEMENT}

This paper was accepted for publication in the Proceedings of the 9th International Conference on Throughlife Engineering Services (TESConf 2020). The definitive published version is available at SSRN at https://ssrn.com/abstract=3717733 or http://dx.doi.org/10.2139/ssrn.3717733.

\section{LICENCE}

CC BY-NC-ND 4.0

\section{REPOSITORY RECORD}

Ariansyah, Dedy, Achim Buerkle, Ali Al-Yacoub, Melanie Zimmer, John Ahmet Erkoyuncu, and Niels Lohse. 2020. "Towards a Digital Human Representation in an Industrial Digital Twin". Loughborough University. https://hdl.handle.net/2134/13286075.v1. 


\title{
Towards a Digital Human Representation in an Industrial Digital Twin
}

\author{
Dedy Ariansyah ${ }^{\mathrm{a}, *}$, Achim Buerkle ${ }^{\mathrm{b}}$, Ali Al-Yacoub ${ }^{\mathrm{b}}$, Melanie Zimmer ${ }^{\mathrm{b}}$, John Ahmet \\ Erkoyuncu $^{\mathrm{a}}$, Niels Lohse ${ }^{\mathrm{b}}$ \\ ${ }^{a}$ Cranfield University, Through-life Engineering Service Centre, Cranfield, MK43 OAL, UK \\ ${ }^{b}$ Loughborough University, Epinal Way, Loughborough, LE11 3TU, UK \\ * Corresponding author. Tel.: +44 (0) 1234 752285; E-mail address: Dedy.Ariansyah@cranfield.ac.uk
}

\begin{abstract}
Digital twins (DTs) have demonstrated their abilities to integrate sensor data, current state information, and the information about the environment in virtual models. While previous approaches have focused on creating DTs for mainly machines and workstations, a small number of studies have considered human performance when designing the DT system, which leads to a deficiency in overall system performance. The absence of the human integrated-DT framework may decelerate human integration in industrial DT, and thus, disregards the crucial role of the human in the industry of the future. This paper presents a framework for digital human representation in an industrial DT to continuously monitor and to analyse the human operational state and behaviour. Thereby, the DT enables decision-makers to allocate tasks on the shop floor taking into account the human physical and mental status. A sample case showed how a human muscle activity monitoring system could be integrated with the DT based on the developed framework to account for the operator's muscular fatigue or physical exhaustion for decisionmaking. This included the use of Artificial Intelligence (AI) to interpret the human activity related data using wearable sensors, such as electromyography (EMG). Future research is proposed to harness human data from a richer variety of sensors as control parameters for production operation and improved decision-making.
\end{abstract}

Keywords: Digital Twin; Digital Human Representation; Electromyography; Machine Learning, Incremental Learning.

\section{Introduction}

In the era of digital technology, data have revolutionised several aspects of our lives, including manufacturing sectors. Hence, the Industry 4.0 initiative has received great attention from the business and research community [1]. Even though the concept is not new and it was within the interest of research for several years under various terms such as, "Industrial Internet" in the USA or "Internet +" in China [2], the term "Industry 4.0 " is now well established in different fields including academia and industry. Some of the key elements of Industry 4.0 are automatic data collection, real-time data monitoring and optimisation [3]. These are important enablers for the creation of cyber-physical systems (CPS) which brings together the physical world and its digital representation to allow more insightful decision-making on the basis of data [4]. The digital representation that reflects the real-time operating conditions of a physical system is generally defined as a Digital Twin (DT) [5]. The elementary concept of DTs is that an object can be represented in a physical and a virtual system. The physical system embodies the real existence of the object, while a virtual system depicts the object using information and models that describe the physical state of the object. Simply put, the virtual systems mirror the state of the equipment through the available information [6]. The bi-directional association of physical equipment with virtual representations can facilitate effective product design, dynamic scheduling of maintenance, decision support systems and systems validations throughout the system's life cycle [7].

The DT concept becomes a critical tool in manufacturing, due to the dynamic change in demand and supply [8]. One of the hot topics in this field is the combination of intelligent sensing systems, Internet of Things (IoT) and DT, as it makes the production of products more efficient and intelligent [4]. As such, DT can enable the transition into Industry 4.0 by supporting the design, development and control of complex systems in manufacturing. Thus, DT can play a significant role in preventive maintenance, task scheduling, resources allocation and decision-support. Even though the human has an essential role within processes such as assembly, maintenance, packaging and many others, existing DT systems rely on the sensor data from equipment without considering the human data. 
Therefore, this paper proposes the DT framework that takes the digital human representation which is the collection of human data and models (e.g. datadriven or mathematical models) to predict human cognitive and physical status, perception, and behaviours. The framework takes advantage of the emergence of technologies such as intelligent sensors, wearable sensors, Artificial Intelligence (AI), communications and computations, as these technologies provide the possibility to monitor the operators' physical and psychological activity on the shop floor. Consequently, the framework integrates the real-time representation of the human state within the DT, so that decision-makers can have a deeper insight into the available resources and human status before allocating tasks. Furthermore, the human integrated DT framework offers the potential to make the workspace more comfortable for the human, since dangerous and distressing situations can be detected, and mitigation in the form of additional resource or different task allocation can be provided. Moreover, the neglected human data as feedback to the DT system hinders the exploitation of human-machine partnership that takes human intelligence and flexibility into account. In seeing the necessity for human integration, there is a need for understanding of human roles in the future industrial context and how the advancement in digital manufacturing technology will change the way humans interact and exchange information with the production system.

The remainder of this paper is structured as follows: In Section 2, a literature review on the topics of DT and human representation are presented. The proposed digital human representation in DT systems is outlined in Section 3. An illustrative example of DT depicting the proposed framework is described in Section 4. Finally, Section 5 presents conclusions and future work.

\begin{tabular}{|ll}
\hline Nomenclature \\
AI & Artificial Intelligence \\
ANN & Artificial Neural Network \\
CPS & Cyber-Physical System \\
DT & Digital Twin \\
EMG & Electromyography \\
HRC & Human-Robot Collaboration \\
IoT & Internet of Things \\
IT & Information Technology \\
ROS & Robot Operating System \\
SVM & Support Vector Machine
\end{tabular}

\section{Literature Review}

\subsection{The Role of the Human in the Future Industrial Context}

Industry in the future aspires a smart ecosystem in which humans, machines, and organisation management systems exchange information and synergise to achieve operational excellence. The strategic initiative, so-called Industry 4.0, which was put forward almost a decade ago foresees IoT and Cyber-Physical System (CPS) as key technologies for the realisation of Smart Factories [10]. In the CPS, all the IoT-enabled devices/machines are interconnected within a networked production system in which intelligent computer algorithms are employed to automatically control the information exchange and to monitor the physical processes. This new generation of 'smart system' enabled by CPS is believed to facilitate the achievement of the fundamental improvements in the industrial processes related to manufacturing, engineering, material usage, supply chain, and lifecycle management [10]. Nevertheless, before fully harnessing the power of CPS, the implementation of the CPS needs to resolve the integration issues related to legacy systems and the ethical aspect regarding the human role [11]. In the context of human integration, the trend of the future industry is foreseen to fall in between two extremes; 'technocentric' or 'anthropo-centric', following the diffusion of CPS [12]. The former implies that industrial operations, human roles, and decisions would be governed by Information Technology (IT), allowing the human to intervene only in the case of malfunction or any other erroneous processing in the system. The latter sees IT as a decision support system to assist a human whose responsibility is to optimise and intervene in the industrial processes. The likelihood or the degree of CPS development route considering the aforementioned scenarios will depend on the companies' choice of the design of the technology and work organisation [11]. The observed tendency from the literature seems to project the exploitation of 'interconnected systems' for enhancing human skills in dealing with a range of problems and complexities rather than controlling and allocating human resources for various demands in the factory $[13,14]$

In Smart Factories, one study showed that the access of structured information from the CPS within production systems significantly supports manufacturing employees in performing daily tasks, which leads to incremental improvement of industrial benefits in terms of time, cost, and process quality [15]. Another example of augmented human capability supported by intelligent personal digital assistance was demonstrated in [16]. In relation to 
the CPS architecture, the other study proposed the SOPHOS-MS framework to design a personal digital assistant system that provides real-time data and Augmented Reality contents as well as the interactive knowledge of manufacturing system in the form of Question \& Answer. The utilisation of this system significantly improves the learning time of training of manufacturing tasks which is much needed especially with the increasing complexity of the manufacturing landscape. Ultimately, the goal of connecting human with CPS is to provide comprehensively understandable information that is easily accessible when in need to avoid any ineffective activities and to enable effective and efficient problem-solving skills [17]. Although many researchers tend to favour CPS as a decision support system for a human task, the propensity toward the other extreme where the human task is communicated and coordinated by the production system is necessary to improve the working environment $[18,19]$

\subsection{The Role of Digital Twin in CPS-based Production System}

In Smart Factories, all elements in the CPS-based production system continuously communicate and exchange the information to improve the overall production system performance. Two main entities on which CPS is built are [20]: (1) the advanced connectivity that ensures accurate data acquisition from the physical world and information feedback from cyberspace; and (2) intelligent data management, analytics, and computational capabilities that constructs the cyberspace. As highlighted, the CPS relies on real-world data and information analytics to extract insight from the network. For example, self-comparison ability which enables the performance of a single machine to be compared to the fleet [20]. This, in turn, provides the benefits for monitoring and optimising the operation of an individual machine.

At this point, however, the CPS is somewhat limited to the prediction capability in terms of examining what-if scenarios. Since the CPS relies on past and present data to predict the future, it cannot examine novel situations or explore a new possibility that provides new value. Another concept of physical-cyber integration that can complement CPS is the DT. DT is a virtual representation of a physical system or a process that relies on the field data and models to simulate the behaviour and provide feedback. Since the DT emulates the physical object in virtual space through the models, various behaviours of its physical counterpart can be captured and simulated to improve decision-making.

Meanwhile, the research trends on determining human involvement in CPS often consider the human as an expert whose task is to make a decision or perform operations based on the acquired knowledge from the cyberspace [20]. Although the decision-making skills and problem-solving capabilities of the employee are potentially improved by the CPS-based support system, there are drawbacks in sustaining the overall system performance. The reason is this paradigm essentially ignores that the overall system performance that involves the human in their operation will degrade when human resources do not match the required task demand. In the light of human integration with CPS, a digital representation of the human enabled by DT can serve as a participant in a CPS-based production system where human capacity (e.g., cognitive and physical) is incorporated in the decision-making to achieve sustainable productivity. By virtually connecting the human into the CPS, every performance aspect of the production system can now be achieved through the interaction of its components by sharing the relevant locally generated information and individual system capabilities. The result is that each participant in the CPS is intelligently enriched and functionally empowered to perform its specified task. The next sub-section describes how capturing human data can be beneficial in adjusting or modifying the human task to maintain the high-level system performance

\subsection{Human Data Collection to complement the DT}

Despite different levels of automation within the manufacturing domain, human performance is still considered to have a significant impact on the overall system performance. Subsequently, it is desirable to monitor factors that affect human task performance and subsequent error potentials [21]. This would allow for maximising human capabilities.

Substantial research into human physiological measures has indicated correlations between the human given tasks and physiological activity. For example, a demanding task could be correlated to an increase in heart rate and change in heart rate variability [22]. This could be interpreted as stress experienced by the human operator. Overall, physiological metrics amass a large variety of sensors, that can be integrated into a DT to measure the human current state, The sensors can be further grouped into psychophysiological and more physical-physiological measurements [23].

Psychophysiological measurements such as electrodermal activity, cardiovascular signals, respiration measurements, brain activity, pupil diameter, and blink rate are often utilised to quantify the mental status of a human operator. This includes measurements of mental workload [24]. Within the manufacturing domain and especially during 
repetitive shift work, human operators are susceptible to mental work overload. The UK Health and Safety Executive states that high levels of mental workloads occur during very complicated or monotonous tasks. This is expected to lead to fatigue, stress and subsequent errors [25]. However, one of the main issues with psychophysiological measurements is that they often lack a single monolithic interpretation. Thus, they often need to be combined with subjective metrics such as selfreports or questionnaires [24]. Yet, studies demonstrated that a combination of different physiological metrics could be tied to achieve more accurate prediction results for human workload [26,27].

Physical physiological measurements, on the other hand, include Muscular and skeletal positioning, as well as muscle tension, which indicates high payloads or muscular fatigue of a human operator [24]. In general, the contraction of a muscle generates electrical activity which can be measured through electrodes. This technique is known as electromyography (EMG).

In a manufacturing context, studies have considered EMG signals to detect excessive physical fatigue, which may result in musculoskeletal disorders [28]. In that case, a robot could then assist to reduce the payload and the subsequent load on human joints [29]. The EMG signals showed an increase in mean amplitude and a decrease of the frequency spectrum during increased muscle fatigue. Yet, it is stated that this method works well for constant muscle effort, while it performs less reliable for dynamic tasks where muscle efforts are undergoing constant changes [28]. Thus, the measured EMG signals were used in a model in conjunction with estimated forces to establish a muscle fatigue model [28]. In the context of the DT, observing and monitoring the current physical and psychophysiological state could help to improve decision-making.

\subsection{Challenges of EMG Data Collection}

In a review on EMG-based continuous human upper-limb motion prediction published in 2019, two main approaches for classifying EMG signals are presented. The approaches are either based on classic Machine Learning methods such as Support Vector Machines (SVMs) or more advanced classifiers such as Artificial Neural Networks (ANNs). The methodologies shown in Fig. 1, typically start with raw EMG data acquisition.

Data Acquisition: As can be seen in Fig. 1, all three methods share this step. Even very advanced classifiers achieve low prediction accuracies if the quality of the EMG signal is poor. Thus, a careful selection of acquisition device and the position of channels above muscles are essential [30]. In nonmedical applications, the term "EMG" is treated as a synonym of "non-invasive EMG", since invasive EMGs would require medical expertise to operate $[24,30]$. Thus, invasive EMGs are not considered as applicable in a manufacturing context.

Feature Extraction: This step often includes preprocessing such as the application of Fourier Transform or Independent Component Analysis to extract certain bandwidths or features mainly responsible for muscular fatigue, while avoiding noise/artefacts. ANNs, however, are typically trained with raw EMG data to identify the relevant constellation of features themselves [30].

Classifier Train Test: For both classic Machine Learning classifiers, as well as ANNs, training and testing are performed before a model can be used online/continuously [28,30].

Online Usage: One of the main issues identified in [30] regarding the classification of EMG data is the deviation between the classification accuracy of offline models when applied online. This is often attributed to subject-specific characteristics within the EMG data, which even occurs during different recording sessions [30,31].

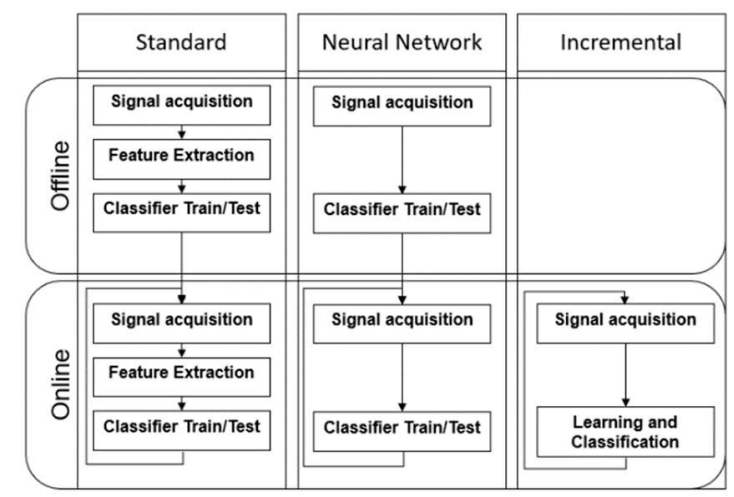

Fig. 1. EMG data processing methodologies.

Incremental Learning: An approach to overcome these limitations can be found in incremental learning. As shown in Fig. 1, the learning and classification occur online only. Moreover, it offers the ability of life-long learning, which allows to incrementally tune the model's structure and thus adapt to subject-specific characteristics within the data [31,32]. Furthermore, no prior knowledge about the data is required such as the number of classes or instances, which allows to significantly lower the manual tuning efforts [10]. However, there are disadvantages to Incremental Learning such as the risk of a plasticity-stability dilemma. This implies that the model is supposed to obtain new knowledge, yet, not forget previous knowledge. Also, the more complex a model becomes, the longer the convergence time will be, which includes classification and performing the learning operation $[31,32]$. Thus, these problems need to be considered 
when applying Incremental Learning for sensory data. Nevertheless, Incremental Learning would allow lowering the manual effort when adding and integrating various physiological sensors to the virtual human model within the DT. Thus, this would allow considering human performance and state within the DT. As an initial sample case, this paper presents an approach based on EMG data in a Human-Robot collaborative task to establish human muscle activity monitoring within the DT. This is intended to further optimise the coaction between humans and robots in the industrial DT to capture and model such interactions. Also, EMG signals have been used in this illustrative example since several researchers have highlighted the benefit of using EMG to predict physical fatigue [33].

\section{Digital Human Representation Framework}

Fig. 2 presents the proposed DT human representation framework that put forward the 'golden mean' (i.e. the desirable middle between two extremes namely, 'techno-centric' and 'anthropo-centric' for human integration) in which the human and the manufacturing technology partnership can be fully realised.

The proposed framework extends the existing three main entities of DT systems [34] namely, physical shop floor, virtual shop floor, and communication network, by specifying elements involved for integrating human in DT systems.

\subsection{Physical shop floor}

In the physical shop floor, there are three main elements which perform different functions: 1) the production element which includes machines, production lines, materials, workers, processes, etc. to produce a product; 2) data perception element which consists of IoT-based sensors, data storage, and communication technology for collecting, saving, and sending the data, status, and behaviours of the production element to the communication network; and 3) feedback interface element which involves a mechanism for the human to access and update virtual shop floor information such as a smart device support system as well as control information/action to regulate the performance and operation of a physical production equipment such controllers and actuators. The result of the feedback element entails the adjustment of production operation to human capability and vice versa to achieve the common goal. For example, the task/event information is the feedback generated by the virtual shop floor to managing task distribution between the equipment and the human operator.

\subsection{Virtual shop floor}

The virtual shop floor is a digital representation of the physical shop floor which consists of two main elements: 1) virtual representation element, which contains integrated models that process the field data to emulate the life of its physical

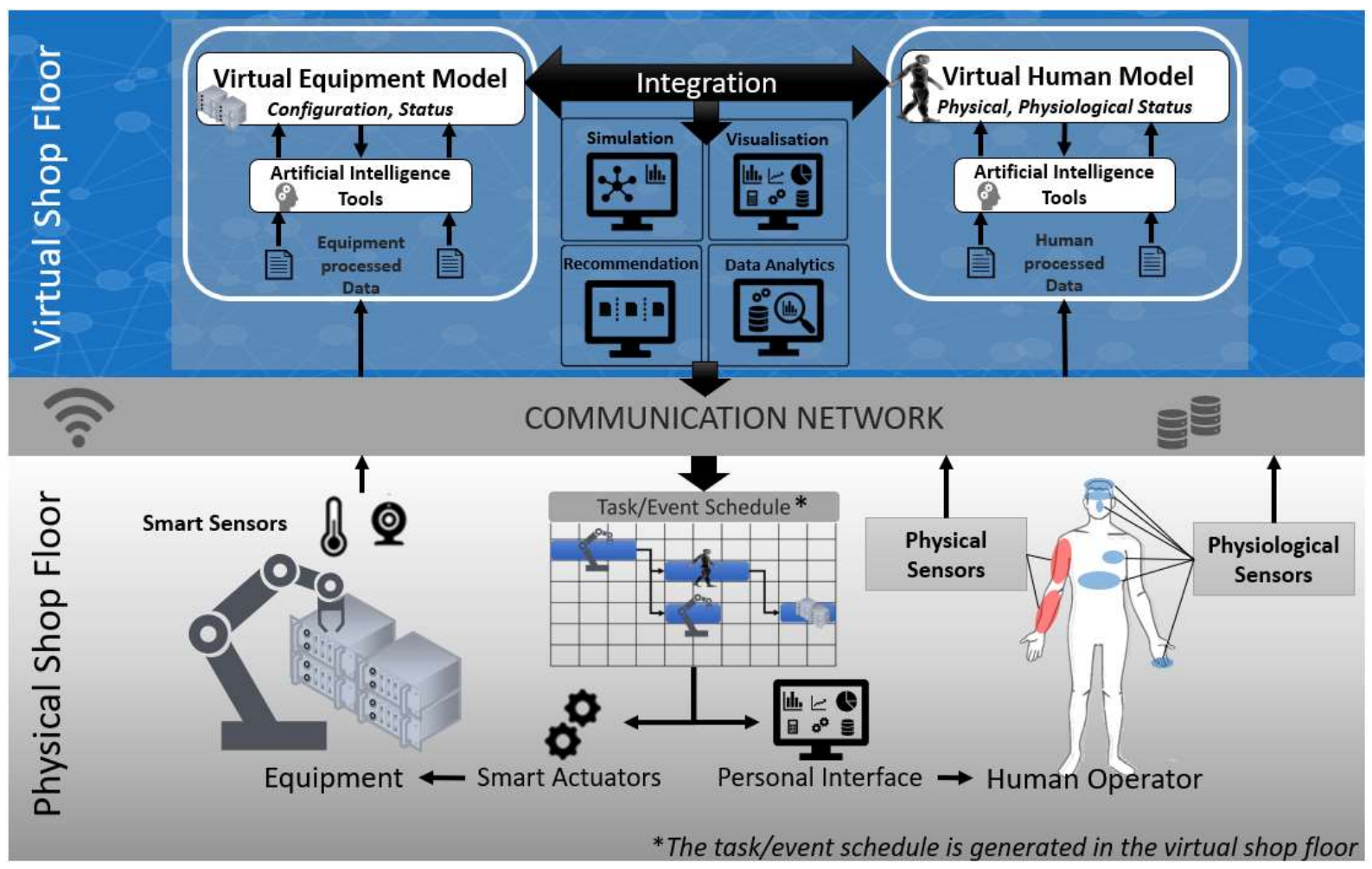

Fig. 2. The proposed human integrated DT framework. 
counterpart (e.g. machine, human, human-machine interaction, etc.). This includes geometrical, physical, behaviour, and rule models for virtual production equipment [35]. Meanwhile, the virtual human and its interactions may include geometrical, psycho-physiological, physical-physiological models, and so on. It can also include AI-based tool to produce data-driven models and/or to compare the correspondences between physical and virtual data for dual optimisation. 2) insight element, which relies on AI-based processing, simulation, data analytics, and semantic modelling to process physical and virtual data for gaining insights for optimisation, recommendation, decision-making, and visualisation. Digital representation can be built in local or cloud storage. Information processing functions need to be embedded to convert raw data coming from physical space into usable data for mapping to the virtual shop floor. The typical processes involved are data acquisition, data preprocessing, data analysis, and data fusion [34].

\subsection{Communication network}

The communication network is a means to exchange information between physical and virtual system. It accommodates two types of interactions: 1) production line and $D T$, which involves live data mapping from physical to virtual space as well as performance optimisation and control from virtual to real space, and 2) human and DT, which involves the digital representation of the human and knowledge capture from physical to virtual space, as well as intuitive insight provision for decision-making from virtual to real space. The network implementation varies from Bluetooth, Ethernet, Wi-fi, etc. depending on the range of required data access and also the network performance e.g. bandwidth and latency (4G vs. 5G).

\subsection{Operation mechanism of the framework}

In the future, human workers will perform tasks alongside machines and robots with limited to no physical barriers on the Physical Shop Floor. This realisation requires interconnection between all objects which enables data exchange and collaboration to achieve common goals. Information about the current operational state of the machines is collected by using smart sensors. While, human data is measured by physical sensors such as EMGs, to measure muscle fatigue and physiological sensors such as ECG (Electrocardiogram) to detect mental workload. All sensor data is sent and processed via the Communication Network and interpreted by different AI tools within the DT. Through the collected data and the virtual model, DT gives the transparency of the current status, behaviours, performance of the human and the equipment on the shop floor. In addition, simulation and data analytics can extract useful insights from data processed by the single or integrated models, which are presented through visualisation in the form of recommendations. One example of DT generated insight is a task/event schedule. This schedule determines which party (Robot, Machine or Human operator) will execute a task. In contrast to the previous DT approaches, this schedule takes the human physical and psychophysiological state into consideration. The schedule is communicated via smart actuators or controllers back to robots and machines, and through personalised human interfaces back to the human. An illustrative example is presented to demonstrate the implementation of the proposed framework on a sample case in the following section.

\section{Illustrative example}

One of the emerging problems in Industry 4.0 involves understanding the role of the human in Human-Robot Collaboration (HRC). To illustrate the conceptualisation of the HRC working paradigm, the proposed framework was applied to model and optimise the coaction between humans and robots in the industrial DT. Fig. 3 depicts the considered co-manipulation task in which an instrumented human and collaborative robot, in this case, a UR10, carry a weight (load) in the workspace (physical layer). While the hardware integration, data collection and training of data-driven models have been completed, the visualisation and quantitative evaluation are currently work-inprogress.

Based on Fig. 3, the physical layer has three groups of sensors, which are robot sensors, contextual sensors, and biometric sensors. The robot sensors collect the data of the robot's internal state

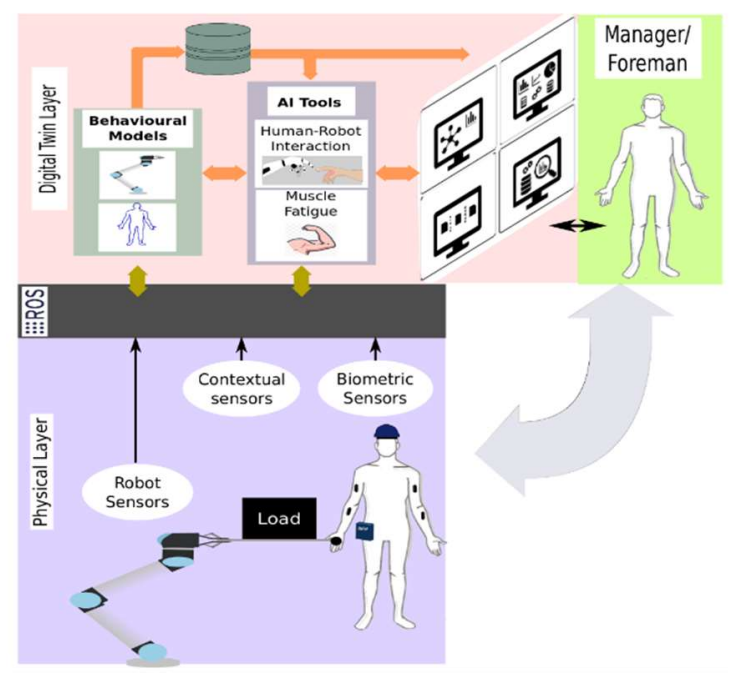

Fig. 3. HRC illustrating the proposed framework. 
such as the joints angles, motor temperature, motor torque, and many others. The biometric sensors measure the physical and psychological state of the human operator such as heart rate, muscle activity EMG signal, head movements, facial temperature, and brainwave signals. The contextual sensors include motion tracking systems and environmental sensors (temperature, humidity, etc.) which collect the data not only on the interactions between the human and the robot but also on their interaction with the surrounding environments. As can be seen, various sensors have been used in this experiment. To integrate them into one network, the Robot Operating System (ROS) was used to enable the data collection during Human-Robot Interaction experiments. The developed ROS package for human wearable sensors has been made publicly available on GitHub for the research community to use [3], and the theoretical foundation of the GitHub tool can be found in [37].

Human cognitive skills are used in navigating the load, while the robot should follow human guidance and carry the weight of the load. The challenge is how to embed an autonomous capability to the robot to safely accommodate to various degree of human physical directions. To achieve this, a force/torque sensor was installed at the robot end effector to give the ability to sense the haptic cues. The robot needs to learn the skill to react to different haptic inputs from different operators. The skill acquisition was done by having two humans (a leader and a follower) perform a co-manipulation task in an unstructured environment.

By collecting displacements, forces and muscle EMG signal, the follower's human behaviour was modelled using a data-driven model, which can be employed and integrated into virtual robot's model to control, simulate, and analyse the required robot behaviour, which takes place in the DT layer. In order to learn a behaviour model of human physical fatigue, an incremental learning approach has been conducted as described in [31]. In light of the proposed DT framework, two behavioural model blocks are shown in Fig 3. The first behavioural block is human follower behaviour; this block represents the control strategy of the robots. This allows us not only to control and simulate the robot behaviour, but it also gives insights on the human operator behaviour during co-manipulation, such as speed and muscle signal. The second behavioural block aims to incrementally learn to predict muscle fatigue in human muscles to notify the operator and manager/foreman about potential upcoming issues. The main advantage of using incremental learning is that the quality of behavioural models is improving over time, and it can adapt quickly to different user data.
The outputs of these blocks will be visualised to managers/foremen who manages the task/event schedule. This task/event then will be communicated to the robot or human stakeholders through controllers and smart actuators as well as personalised human interfaces, respectively. In this example, the identified muscle fatigue of the operator could signal to the robot that additional support needs to be provided to the human to prevent harm. Alternatively, the signal can be sent to inform the operator to take a break or alert the foreman in charge to make arrangements for a temporary substitute. Thus, human operators can be replaced prior to potential injury.

The communication between the physical and the DT layer was achieved via the use of ROS, which provides data structures that are suitable for physical and biometric messages. ROS plays the role of middleware that facilitates the communication and the data exchange between the physical layer and the DT layer. The physical connection between these entities has been realised through $\mathrm{Wi}-\mathrm{Fi}$ and ethernet, in which ROS was the software backbone that allows all these entities to communicate with each other. The exchange of data has been performed using ROS default messages for robot information, while customised messages have been used for human biological data. Also, ROS provides essential functionalities to collect, synchronise and filter data.

\section{Conclusions and Future Work}

This work presents a framework for human digital representation in an industrial DT. The framework extends the existing three main entities of DT framework by specifying design elements in human-integrated DT framework. This is especially critical in an environment where human plays a significant role to achieve system productivity. An illustrative example is presented to show how human integration in an industrial DT is implemented using the developed framework.

While the human data integration can potentially enhance the performance of the DT system to be more adaptive to the human context, the ethical issues around data acquisition, data processing, and so on have yet to be addressed. In the future, more research in human DT integration regarding automatic human data collection, data processing, and human-based data-driven in DT for optimising the whole system performance is required to evaluate the working procedure of the proposed DT framework. Since the proposed framework did not touch upon communication drawbacks such as latency, delays and cloud computations; these limitations will be analysed and considered in the future implementations. 


\section{Acknowledgements}

This work was funded by the EPSRC as part of the "Digital Toolkit for optimisation of operators and technology in manufacturing partnerships" project (DigiTOP; https://digitop.ac.uk; EP/R032718/1).

\section{References}

[1] Carvalho N, Chaim O, Cazarini E, Gerolamo M. Manufacturing in the fourth industrial revolution: A positive prospect in sustainable manufacturing. Procedia Manuf 2018;21:671-8.

[2] Wang S, Wan J, Li D, Zhang C. Implementing Smart Factory of Industrie 4.0: An Outlook. Int J Distrib Sens Networks 2016;2016.

[3] Uhlemann THJ, Lehmann C, Steinhilper R. The Digital Twin: Realizing the Cyber-Physical Production System for Industry 4.0. Procedia CIRP 2017;61:335-40.

[4] Dombrowski U, Wullbrandt J, Krenkel P. Industrie 4.0 in production ramp-up management. Procedia Manuf., vol. 17, 2018, p. 1015-22.

[5] Rosen R, von Wichert G, Lo G, Bettenhausen KD. About the importance of autonomy and digital twins for the future of manufacturing. 15th IFAC Symp. Inf. Control Probl. Manuf., 2015, p. 567-572.

[6] Grieves M. Digital Twin: Manufacturing Excellence through Virtual Factory Replication 2014.

[7] Schleich B, Anwer N, Mathieu L, Wartzack S. Shaping the digital twin for design and production engineering. CIRP Ann 2017;66:141-4.

[8] Tao F, Sui F, Liu A, Qi Q, Zhang M, Song B, et al. Digital twin-driven product design framework. Int $\mathrm{J}$ Prod Res 2019;57:3935-53.

[9] He B, Bai K-J. Digital twin-based sustainable intelligent manufacturing: a review. Adv Manuf 2019:1-21.

[10] Kagermann H, Wahlster W, Helbig J. Securing the future of German manufacturing industry: Recommendations for implementing the strategic initiative INDUSTRIE 4.0. Final Rep Ind 40 Work Gr 2013:1-84.

[11] Energetics Incorporated. Foundations for Innovation in Cyber-Physical Systems: Workshop Report. Columbia, Maryland: 2013.

[12] Dworschak B, Zaiser H. Competences for cyber-physical systems in manufacturing-First findings and scenarios. Procedia CIRP 2014;25:345-50.

[13] Gorecky D, Schmitt M, Loskyll M, Zühlke D. HumanComputer-Interaction in the Industry 4.0 Era 2014:289-94.

[14] Hermann M, Pentek T, Otto B. Design principles for industrie 4.0 scenarios. Proc Annu Hawaii Int Conf Syst Sci 2016;2016-March:3928-37.

[15] Longo F, Nicoletti L, Padovano A. Ubiquitous knowledge empowers the Smart Factory: The impacts of a Serviceoriented Digital Twin on enterprises' performance. Annu Rev Control 2019;47:221-36.

[16] Longo F, Nicoletti L, Padovano A. Smart operators in industry 4.0: A human-centered approach to enhance operators' capabilities and competencies within the new smart factory context. Comput Ind Eng 2017;113:144-59.

[17] Ariansyah D, Rosa F, Colombo G. Smart maintenance: A wearable augmented reality application integrated with CMMS to minimize unscheduled downtime. Comput Aided Des Appl 2020;17:740-51.
[18] Graessler I, Poehler A. Integration of a digital twin as human representation in a scheduling procedure of a cyber-physical production system. IEEE Int. Conf. Ind. Eng. Eng. Manag., Singapore: 2018, p. 289-93.

[19] Graessler I, Poehler A. Intelligent control of an assembly station by integration of a digital twin for employees into the decentralized control system. Procedia Manuf 2018;24:1859.

[20] Lee J, Bagheri B, Kao H-AA. A Cyber-Physical Systems architecture for Industry 4.0-based manufacturing systems. Manuf Lett 2015;3:18-23.

[21] Cheng $\mathrm{H}, \mathrm{Xu}$ W, Ai Q, Liu Q, Zhou Z, Pham DT. Manufacturing Capability Assessment for Human-Robot Collaborative Disassembly Based on Multi-Data Fusion. Procedia Manuf 2017; 10:26-36.

[22] Young MS, Brookhuis KA, Wickens CD, Hancock PA. State of science: mental workload in ergonomics. Ergonomics 2015;58:1-17.

[23] Wastell DG, Newman M. Stress, control and computer system design: A psychophysiological field study? Behav Inf Technol 1996;15:183-92.

[24] Lazar J, Feng JH, Hochheiser H. Measuring the human. In: Lazar J, Feng JH, Hochheiser HBT-RM in HCI (Second E, editors. Res. Methods Human-Computer Interact. 2nd ed., Boston: Morgan Kaufmann; 2017, p. 369-409.

[25] Health and Safety Executive. Manag Shiftw 2018.

[26] Ogorodnikova O. Human Weaknesses and strengths in collaboration with robots. Period Polytech Mech Eng 2008;52:25-33.

[27] Steinfeld A, Fong T, Kaber D, Lewis M, Scholtz J, Schultz A, et al. Common Metrics for Human-Robot Interaction. Assoc Comput Mach 2006.

[28] Peternel L, Fang C, Tsagarakis N, Ajoudani A. A selective muscle fatigue management approach to ergonomic humanrobot co-manipulation. Robot Comput Integr Manuf 2019;58:69-79.

[29] Peternel L, Tsagarakis N, Caldwell D, Ajoudani A. Robot adaptation to human physical fatigue in human-robot comanipulation. Auton Robots 2018;42:1011-21.

[30] Bi L, Feleke A, Guan C. A review on EMG-based motor intention prediction of continuous human upper limb motion for human-robot collaboration. Biomed Signal Process Control 2019;51:113-27.

[31] Buerkle A, Al-Yacoub A, Ferreira P. An Incremental Learning Approach for Physical Human-Robot Collaboration. 3rd UK-RAS Conf. Univ. Lincoln, April 2020, 2020, p. 5-7.

[32] Bouchachia A, Gabrys B, Sahel Z. Overview of some incremental learning algorithms. IEEE Int Conf Fuzzy Syst 2007.

[33] Lariviere C, Arsenault AB, Gravel D, Gagnon D, Loisel P. Evaluation of measurement strategies to increase the reliability of EMG indices to assess back muscle fatigue and recovery. J Electromyogr Kinesiol 2002;12:91-102.

[34] Zheng Y, Yang S, Cheng H. An application framework of digital twin and its case study. J Ambient Intell Humaniz Comput 2019; 10:1141-53.

[35] Tao F, Zhang M, Liu Y, Nee AYC. Digital twin driven prognostics and health management for complex equipment. CIRP Ann 2018;67:169-72.

[36] Intelligent Automation Centre. Blue-box: ROS package for human wearable sensors 2020. https://github.com/IntelligentAutomation-Centre/bluebox.

[37] Al-Yacoub A, Buerkle A, Flanagan M, Ferreira P, Hubbard E-M, Lohse N. Effective Human-Robot Collaboration Through Wearable Sensors. 25th IEEE Conf. Emerg. Technol. Fact. Autom., Vienna, Austria: 2020. 